\title{
In Vivo Human Choroidal Vascular Pattern Visualization Using High-Speed Swept-Source Optical Coherence Tomography at $1060 \mathrm{~nm}$
}

\author{
Reza Motaghiannezam, ${ }^{1}$ Daniel M. Schwartz, ${ }^{2}$ and Scott E. Fraser ${ }^{1}$
}

Purpose. To investigate the retinal and choroidal vascular pattern, structure, and thickness using high-speed, high axial resolution, swept-source optical coherence tomography (SSOCT) at $1060 \mathrm{~nm}$, demonstrating enhanced penetration through all choroidal layers.

Methods. An ophthalmic SS-OCT system was developed operating at 57,000 A-lines/s with $5.9 \mu \mathrm{m}$ axial resolution and was used to collect $3 \mathrm{D}$ images with scanning angles up to $\sim 70^{\circ}$ $\times 35^{\circ}$. The similar features were observed in the choroidal layers by imaging three healthy volunteers. En face images, extracted at different depths, capture features of the retinal and choroidal vasculature networks and substructure. Retinal and choroidal thicknesses were measured over scanning angles of $\sim 14^{\circ} \times 14^{\circ}$, yielding retinal and choroidal thickness maps.

REsults. The retinal layers, choriocapillaris (CC), Sattler's layer (SL), Haller's layer (HL), and the lamina suprachoroid layer (LSL) were delineated in 2D sagittal tomograms. The sagittal tomograms and en face reflectance images over a $2-\mathrm{cm}^{2}$ field of view captured the paraoptic, lateral and medial distal short posterior ciliary artery (SPCA) branches as well as the two lateral and medial long posterior ciliary arteries (LPCAs). En face images in the HL revealed the superotemporal, inferotemporal, superonasal, and inferonasal major choroidal vessels.

Conclusions. High-speed, high-resolution SS-OCT centered at $1060 \mathrm{~nm}$ enables retinal and choroidal vasculature networks visualization, including retina vessels, posterior ciliary artery (PCA) branches, and venous vascular patterns. This technology offers diagnostic opportunities by monitoring change in these networks, substructure, and retinal and choroidal thicknesses during disease initiation and progression. (Invest Opbthalmol Vis Sci. 2012;53:2337-2348) DOI:10.1167/iovs.11-7823

1terations of the choroidal circulation may play important
roles in ocular diseases such as age-related macular
degeneration (AMD), ${ }^{1}$ glaucoma, ${ }^{2}$ and anterior ischemic optic

From the ${ }^{1}$ Beckman Institute, Division of Biology, California Institute of Technology, Pasadena, California; and the ${ }^{2}$ Department of Ophthalmology, University of California and Veterans Affairs Medical Center, San Francisco, California.

Supported by the California Institute for Regenerative Medicine (CIRM), Beckman Initiative for Macular Research, and Caltech Beckman Institute.

Submitted for publication May 2, 2011; revised October 20 and December 16, 2011, and February 8, 2012; accepted February 13, 2012.

Disclosure: R. Motaghiannezam, P; D.M. Schwartz, P; S.E. Fraser, $\mathrm{P}$

Corresponding author: Reza Motaghiannezam, Beckman Institute, Division of Biology, California Institute of Technology, 1200 East California Boulevard, Pasadena, CA 91125; rmotaghi@caltech.edu. neuropathy (AION). ${ }^{3}$ The posterior ciliary artery (PCA) and venous systems are critical components of the outer retinal and optic nerve head $(\mathrm{ONH})$ circulation. The end-arterial nature of the PCA vascular bed and watershed zone sites can be used to explain the extent and pattern of ischemic disorders of the $\mathrm{ONH}$ and choroid. ${ }^{4}$ Although color fundus photography (CF) and fluorescein angiography (FA) are the gold standard methods for retinovascular imaging, ${ }^{5}$ these $2 \mathrm{D}$ imaging techniques lack depth information and deep choroidal blood vessel visualization. Indocyanine green angiography (ICGA) is a better tool for imaging the deeper choroidal vessels, ${ }^{5}$ but, like FA, it is $2 \mathrm{D}$ and requires intravenous injection of a contrast dye. ${ }^{6,7}$ There is a need for noninvasive imaging systems to visualize these key elements in the retinal and choroidal blood circulation.

Ophthalmic optical coherence tomography (OCT) has been demonstrated as a noninvasive depth-resolved technology that allows imaging of the $3 \mathrm{D}$ retina structure in vivo ${ }^{8,9}$ at a resolution approaching that of histologic examination. ${ }^{10,11}$ Current clinical ophthalmic OCT systems use light in the 800$\mathrm{nm}$ wavelength range, which offers limited penetration into the choroid and ONH. ${ }^{12}$ To improve penetration depth and delineate the choroidal structure, an OCT system centered within the 1060-nm water transmission window is described by Unterhuber and co-workers. ${ }^{12,13} \mathrm{~A}$ state of the art ophthalmic high-speed spectral domain (SD)-OCT in the $1-\mu \mathrm{m}$ wavelength range has validated its ability to visualize the choroidal vasculature over $1 \mathrm{~cm}^{2}$ using a novel linear InGaAs diode array camera. ${ }^{14}$

Swept-source (SS)-OCT is a Fourier domain depth-resolved method distinct from SD-OCT. SD-OCT employs a spectrometer and an array of diodes to capture interferograms of a broadband light reflected back from the sample; in contrast, SS-OCT uses a single photodiode detector to resolve time-spectral encoded information in the backscattered light of a laser source that rapidly scans its wavelength. SS-OCT offers several potential advantages over SD-OCT, including increased sensitivity through the full imaging depth, decreased fringe washout, better axial resolution over a broad imaging range, and higher detection efficiencies. ${ }^{15}$ These differences may be even more pronounced in imaging the eyes of patients with media opacities such as cataract. A clinical high-speed SS-OCT system with enhanced penetration would offer the potential to monitor the choroidal vasculature structure, to map retinal and choroidal thicknesses, and to investigate the individual elements of the blood supply during initiation and progression of ocular diseases. Research grade instruments implementing $1-\mu \mathrm{m}$ SS-OCT have been demonstrated with scan rates up to $249 \mathrm{kHz}^{16-19}$ A $28 \mathrm{kHz}$ Santec swept laser source ( $72 \%$ duty cycle) has been employed for $3 \mathrm{D}$ choroidal visualization in a normal subject using scattering optical coherence angiography method. ${ }^{18}$

Here, we report an in vivo $57-\mathrm{kHz}$ SS-OCT centered at 1060 $\mathrm{nm}$ (1013-1103) and demonstrate its use for imaging normal human choroidal vascular anatomy. This SS-OCT system has 
axial resolution of $\sim 5.9 \mu \mathrm{m}$ in tissue and $\sim 101 \mathrm{~dB}$ sensitivity (1.2 $\mathrm{mW}$ incident power). We used this system to image the 3D structure of the human retinal/choroidal vasculature with scanning angles up to $70^{\circ} \times 35^{\circ}\left(2-\mathrm{cm}^{2}\right.$ field of view $)$. Depthintegrated en face images extracted from different depths clearly capture intraretinal layers and choroidal substructures. Using our prototype SS-OCT system, we were able to visualize the lateral and medial PCAs as well as major draining choroidal veins, and we could image clearly their entrance sites, branching locations, coursing directions, and sizes. We captured the similar features by imaging the different choroidal layers of three healthy volunteers. The retinal and choroidal thicknesses were measured and compared in these three subjects. The repeatability of retinal and choroidal thickness measurements was examined in one of the subjects.

\section{Methods}

\section{$1060 \mathrm{~nm}$ SS-OCT System}

The SS-OCT system was comprised of a polygon-based swept-laser source, ${ }^{20}$ an interferometer, and a data acquisition (DAQ) unit (Fig. 1). The swept laser source was constructed in a linear-cavity configuration using a broad-bandwidth semiconductor optical amplifier as a gain medium. ${ }^{17}$ One port of the amplifier was coupled to a wavelengthscanning filter, comprised a collimator with a $1 / \mathrm{e}^{2}$ beam width of 3 $\mathrm{mm}$, a diffraction grating, a telescope, and a polygon mirror scanner. Each facet of the polygon mirror generated a single sweep of the laser output ranging from 1013 to $1103 \mathrm{~nm}$ as it rotated through the beam path of the telescope. To achieve long photon intracavity lifetime, the resonator air-spaced length was reduced to $<1 \mathrm{~m}$. The gain level of the intracavity semiconductor optical amplifier (SOA) and the rotational rate of the polygon were adjusted to provide an average power of $\sim 8$

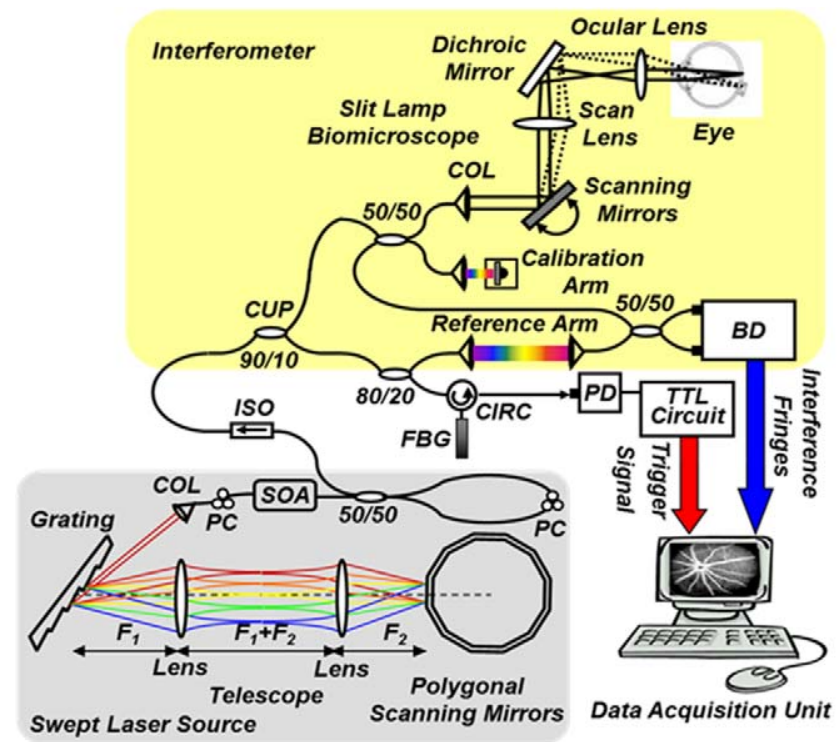

FiguRE 1. Schematic of a polygon-based swept-laser source (gray box), interferometer (yellow box), and SS-OCT data acquisition unit used for imaging. SOA, semiconductor optical amplifier; diffraction grating (830 lines $/ \mathrm{mm})$; telescope $\left(f_{1}=40 \mathrm{~mm}, \mathrm{f}_{2}=35 \mathrm{~mm}\right)$; polygonal scanning mirrors ( 72 facets); COL, collimator; PC, polarization controller; ISO, isolator; CIRC, circulator; CUP, coupler; PD, photodetector; BD, dual balanced photodetector (InGaAs, $80 \mathrm{MHz}$ ); FBG, fiber Bragg grating $(0.1 \mathrm{~nm})$. Data acquisition unit was comprised of an $\mathrm{AD}$ conversion board (14-bit, GaGe CompuScope 14,200) for digitizing interference fringes and a D/A board (16 bit, National Instruments) for driving scanning mirrors (Cambridge Technology).
$\mathrm{mW}$ over the 90-nm full-width sweep bandwidth at a 57-kHz repetition rate and an approximately $100 \%$ unidirectional sweep.

The swept-source output was coupled to the interferometer through an isolator where a $90 / 10$ coupler was used to split light into a sample arm and reference arm. The sample arm light was split equally between the calibration arm and a slit lamp biomicroscope (Carl Zeiss Meditec) as shown in Figure 1. A 50/50 coupler combined and directed the reflected light from the sample to the one port of the interferometer output coupler. The reference arm light passed through a pair of collimators and was directed to the second port of the interferometer output coupler. Resulting interference fringes were detected on both output ports using a dual balanced photodetector. To generate a trigger signal at the beginning of the first interference fringe for the $\mathrm{A} / \mathrm{D}$ conversion board, a portion of the reference arm light was directed through a three-port circulator to a fiber Bragg grating to generate an optical pulse that was detected by a photodetector and converted into an electronically tunable TTL signal. A D/A board was used to generate the driving signals of the two-axis galvanometers, with a user interface and data acquisition control provided by LabView. The SS-OCT system performance specifications were experimentally characterized by measuring the point-spread function using a calibrated partial reflector through imaging depth in air. The sensitivity was measured at $\sim 101 \mathrm{~dB}$ at $100 \mu \mathrm{m}$ delay for an incident power of 1.2 $\mathrm{mW}$ with $5.2-\mathrm{mm}$ imaging range and $8-\mu \mathrm{m}$ axial resolution (equivalent to $5.9 \mu \mathrm{m}$ in tissue by a computational group refractive index of $\mathrm{n}=$ 1.35). The $57-\mathrm{kHz}$ SS-OCT system had $6 \mathrm{~dB}$ of sensitivity roll-off at 1.6 $\mathrm{mm}$. Axial resolution did not degrade with depth across a 3-mm imaging range in air.

\section{In Vivo Vasculature Visualization in Normal Subjects and Scanning Protocols}

The prototype SS-OCT instrument was used to image the eyes of three healthy volunteers (19-year-old Caucasian male, 37-year-old Caucasian male, and 28-year-old Asian female) at the California Institute of Technology (Caltech). This study was approved by the Caltech committee for the protection of human subjects and adhered to the tenets of the Declaration of Helsinki. Written informed consent was obtained from subjects prior to OCT imaging. Total exposure time and incident exposure level were kept less than 5.5 seconds and $1.2 \mathrm{~mW}$ in each imaging session, consistent with the safe exposure determined by American National Standards Institute (ANSI) and International Commission on Non-Ionizing Radiation Protection (ICNIRP). ${ }^{21,22}$ Patient interfaces were based on Stratus OCT-3 system (Carl Zeiss Meditec) adapted with optics to support the 1060-nm wavelength range. A 60-D lens was used at the exit of the fundus camera with 13$\mathrm{mm}$ working distance, providing a beam diameter of either $1.1 \mathrm{~mm}$ on the cornea $(\sim 20 \mu \mathrm{m}$ transverse resolution for SS-OCT imaging) or 1.5 $\mathrm{mm}$ on the cornea ( $\sim 15 \mu \mathrm{m}$ transverse resolution). Several scanning protocols were implemented (Table 1). A 2D protocol generated horizontal tomograms with 907 depth scans spanning $10 \mathrm{~mm}$ in 0.02 seconds. A number of 3D protocols consisted of 500 frames (B-scans) with 453 depth scans (A-scans) per frame, acquired in 5 seconds. In all scanning protocols, the fast scan axis was sagittal (superior-inferior) and the slow axis was horizontal (nasal-temporal). 3D data sets $(5 \times 5$ $\mathrm{mm}$ ) were acquired over the perifovea or over the ONH. Larger 3D data sets $(10 \times 10 \mathrm{~mm})$ were obtained over the lateral side of the fovea (including the fovea) or over the medial side of the ONH (including $\mathrm{ONH})$. In the study of feature reproducibility between subjects and among thickness measurements, 3D data sets were acquired over the perifovea $(4 \times 4 \mathrm{~mm})$ at $15-\mu \mathrm{m}$ transverse resolution.

\section{Image Processing}

The digitized signals were divided into individual spectral sweeps in the postprocessing algorithm. Equal sample spacing in wave number (k) was achieved using a calibration trace at $1.5-\mathrm{mm}$ interferometer delay and numerical correction of the nonlinearly swept waveforms. ${ }^{23}$ 
TABLE 1. Implemented scanning protocols for retinal and choroidal vasculature visualization in three healthy volunteers

\begin{tabular}{lclcc}
\hline Protocol \# & $\begin{array}{c}\text { Protocol } \\
\text { Type }\end{array}$ & \multicolumn{1}{c}{ Scanning Region } & $\begin{array}{c}\text { Scanning Range or } \\
\text { Field of View }(\mathbf{m m})\end{array}$ & $\begin{array}{c}\text { Transverse } \\
\text { Resolution }(\boldsymbol{\mu m})\end{array}$ \\
\hline 1 & 2D & Across the fovea centralis, paraoptic, ONH, perifovea, fovea, and ONH & 10 & 20 \\
2 & 3D & Perifovea & $5 \times 5$ & 20 \\
3 & 3D & Peripapillary & $5 \times 5$ & 20 \\
4 & 3D & Lateral side of the fovea (including fovea) & $10 \times 10$ & 20 \\
5 & 3D & Medial side of the ONH (including ONH) & $10 \times 10$ & 20 \\
6 & 3D & Perifovea & $4 \times 4$ & 15 \\
\hline
\end{tabular}

Image background subtraction and numeric compensation for second order dispersion ${ }^{24}$ were performed. The complex Ss-OCT data sets were upsampled by a factor of 4 and Fourier transformed. Axial motion correction was achieved on the obtained SS-OCT data by cross correlating the consecutive horizontal tomograms. Mean filtering was performed on four consecutive horizontal tomograms, increasing signal to noise and decreasing speckle to enhance 2D tomogram visualization.

The 3D OCT data sets were visualized as depth-integrated en face images, summing the reflectance signal over a selected depth region of 3D data sets. The visualized depth region was either a fixed thickness of tissue in a layer or a selected retina layer after the contours of the different retina layers and Bruch's membrane were detected using an automatic segmentation algorithm. ${ }^{25}$ Since the thickness and depth location of a choroidal layer vary between different subjects, the density of hyperreflective melanocytes as well as vessels size and density were used to differentiate choroidal layers in each subject's eye, as previously described by Považay et al. ${ }^{14}$ Each choroidal region (thickness and depth location) was manually determined and quantified using these qualitative analyses and detecting the choroidal-scleral junction. ${ }^{26}$ In the image renderings for the second subject, we employed the color-coding method proposed by Považay et al. ${ }^{14}$ to signify depth locations of the en face images. The thicknesses of the depth regions are indicated in the legends. To map the retinal

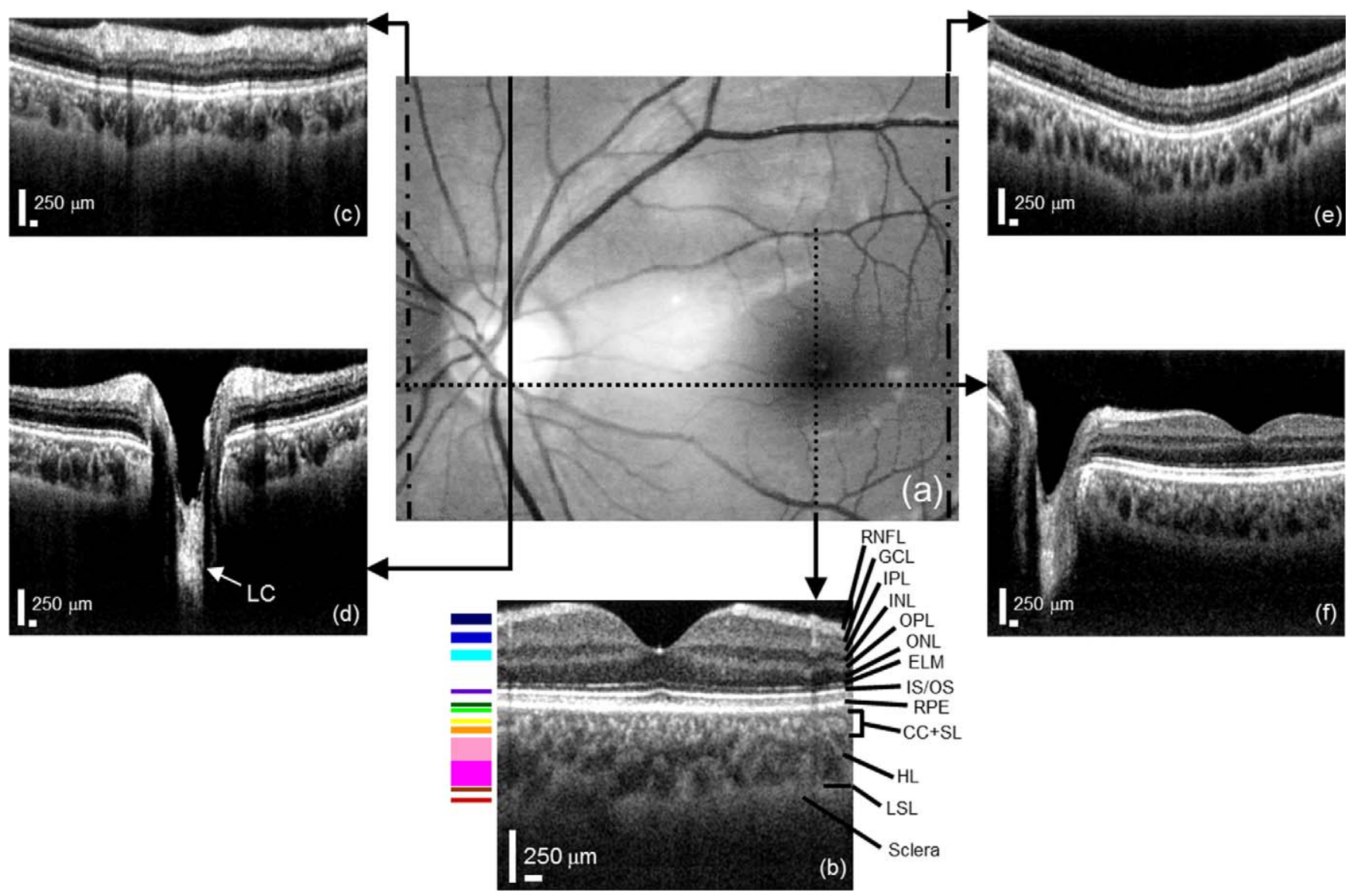

FIGURE 2. (a) Fundus image of a 37-year-old normal subject's right eye acquired across the perifovea and paraoptic regions. (b) SS-OCT 2D sagittal tomogram across the fovea centralis (dotted line, $5 \mathrm{~mm}$ ) exhibiting the nerve fiber layer (NFL), ganglion cell layer (GCL), inner plexiform layer (IPL), inner nuclear layer (INL), outer plexiform layer (OPL), outer nuclear layer (ONL), external limiting membrane (ELM), inner/outer photoreceptor segments (IS/OS), and retina pigment epithelium (RPE). Choroidal region in this tomogram showing choroidal structures including: choriocapillaris (CC), Sattler's layer (SL) with medium-sized blood vessels, and Haller's layer (HL) with large vessels as well as the avascular lamina suprachoroid layer (LSL). SS-OCT 2D sagittal tomograms across (c) the paraoptic (dash-dot line, $10 \mathrm{~mm}$ ), (d) the ONH (solid line, $10 \mathrm{~mm}$ ), and (e) the perifovea (dotdot-dash line, $10 \mathrm{~mm}$ ). SS-OCT 2D transverse tomogram across (f) the fovea and ONH (dashed line, $10 \mathrm{~mm}$ ) reaching the lamina fusca sclerae (LFS). Higher (lower) reflectivity is represented by white (black) in the grayscale. Color-coding in (b) shows the depth locations of each en face image shown in Figures 3-9 (signified by the surrounding box colors). White arrow indicates the lamina cribrosa (LC) in (d). 

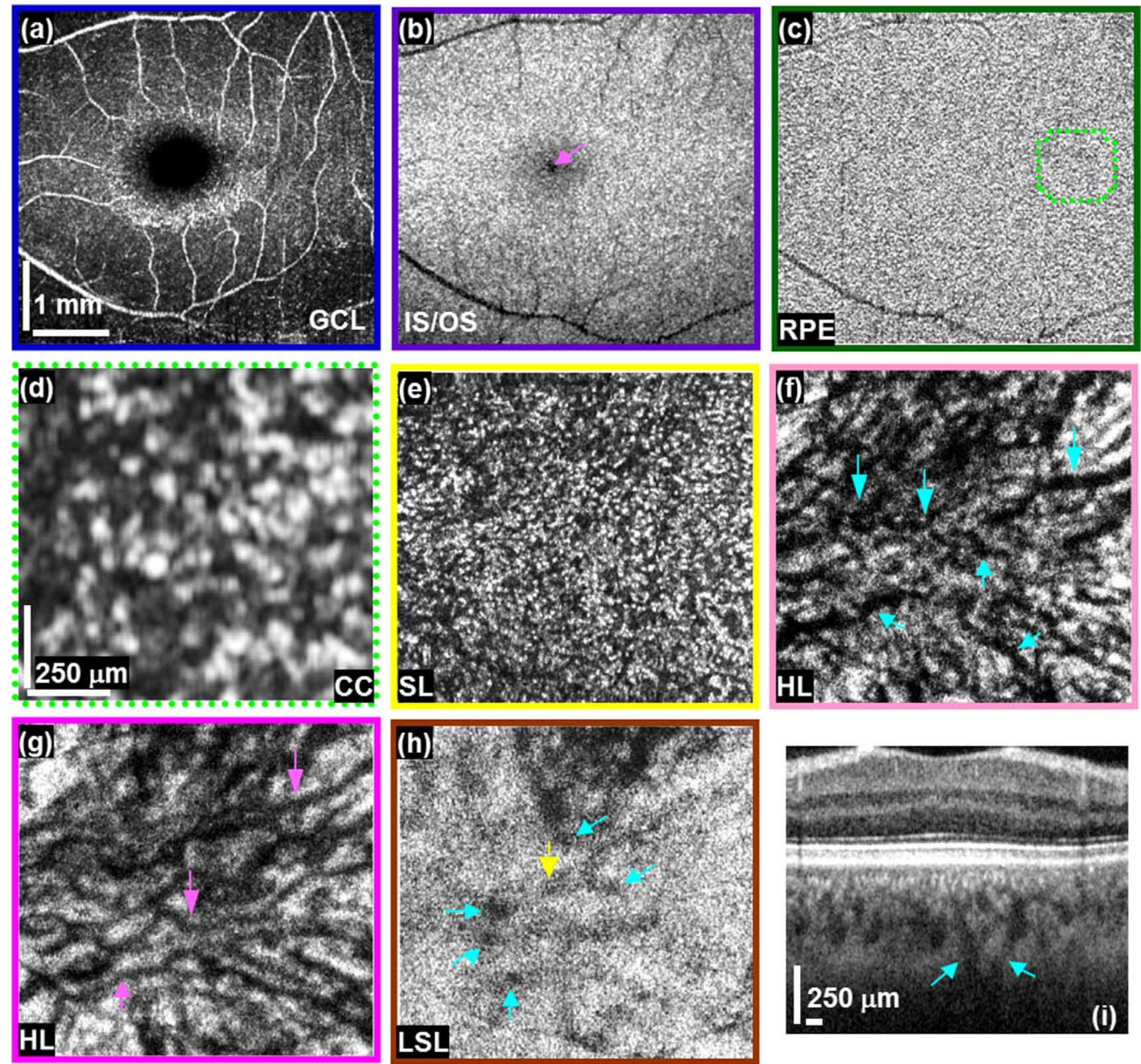

FigURE 3. SS-OCT perifoveal en face views of the retinal and choroidal substructures $(5 \times 5 \mathrm{~mm})(\mathbf{a}-\mathbf{h})$ and temporal distal SPCA visualization in a SS-OCT 2D sagittal tomogram (i) in a 37-year-old normal subject's right eye. (a) The superficial blood vessels in the GCL. (b) The IS/OS en face view capturing the photoreceptors and the fovea centralis (magenta arrow). (c) The spotted appearance of the RPE. (d) The captured spotted appearance at the level of CC (thickness $=20 \mu \mathrm{m}$ ) beneath $1 \mathrm{~mm}^{2}$ RPE (signified with dotted line in c). (e) Arterioles and venuoles (dark spots) as well as hyperreflective melanocytes of the SL (thickness $=20 \mu \mathrm{m}$ ). (f) The inner HL (thickness $=50 \mu \mathrm{m}$ ) with straight and tortuous vessels $(c y a n$ arrows). (g) The larger lumen vessel branches coursing straightly (magenta arrows) in the outer HL (thickness $=50 \mu \mathrm{m}$ ). (h) The entrance sites of the temporal distal SPCAs (cyan arrows) and the fovea centralis projection (yellow arrow) at the level of LSL (thickness $=20 \mu \mathrm{m}$ ). (i) The sagittal tomogram depicting the temporal distal SPCAs (cyan arrows) piercing the LSL.

thickness, the distance between the detected vitreoretinal interface and Bruch's membrane contour was calculated. The choroidal thickness map was generated by calculating the distance between Bruch's membrane contour and the choroidal-scleral junction using smoothing methods ${ }^{26}$ between five slices $(50 \mu \mathrm{m})$ and polynomial curve fitting in both transverse directions. Physiological depths and thicknesses were scaled in the retina and choroid by a computational group refractive index of $n=1.35$ and $n=1.4$, respectively. ${ }^{26}$ To study the repeatability of retinal and choroidal thickness measurements in a normal subject, four independent OCT data sets were acquired over an hour period of the macular region of the 37-year-old subject's right eye (second subject). Retinal and choroidal thickness maps were generated for each data set after data registration, providing a mean and standard deviation for each location. In some of the 3D data sets, arteries were distinguished from veins in the HL by tracing the branches of the PCA.

\section{Results}

\section{Wide-Angle Retinal and Choroidal Layers Delineation}

Figure 2a shows a fundus image of a 37-year-old normal subject's right eye. Figure $2 \mathrm{~b}$ presents a sagittal foveal tomogram 


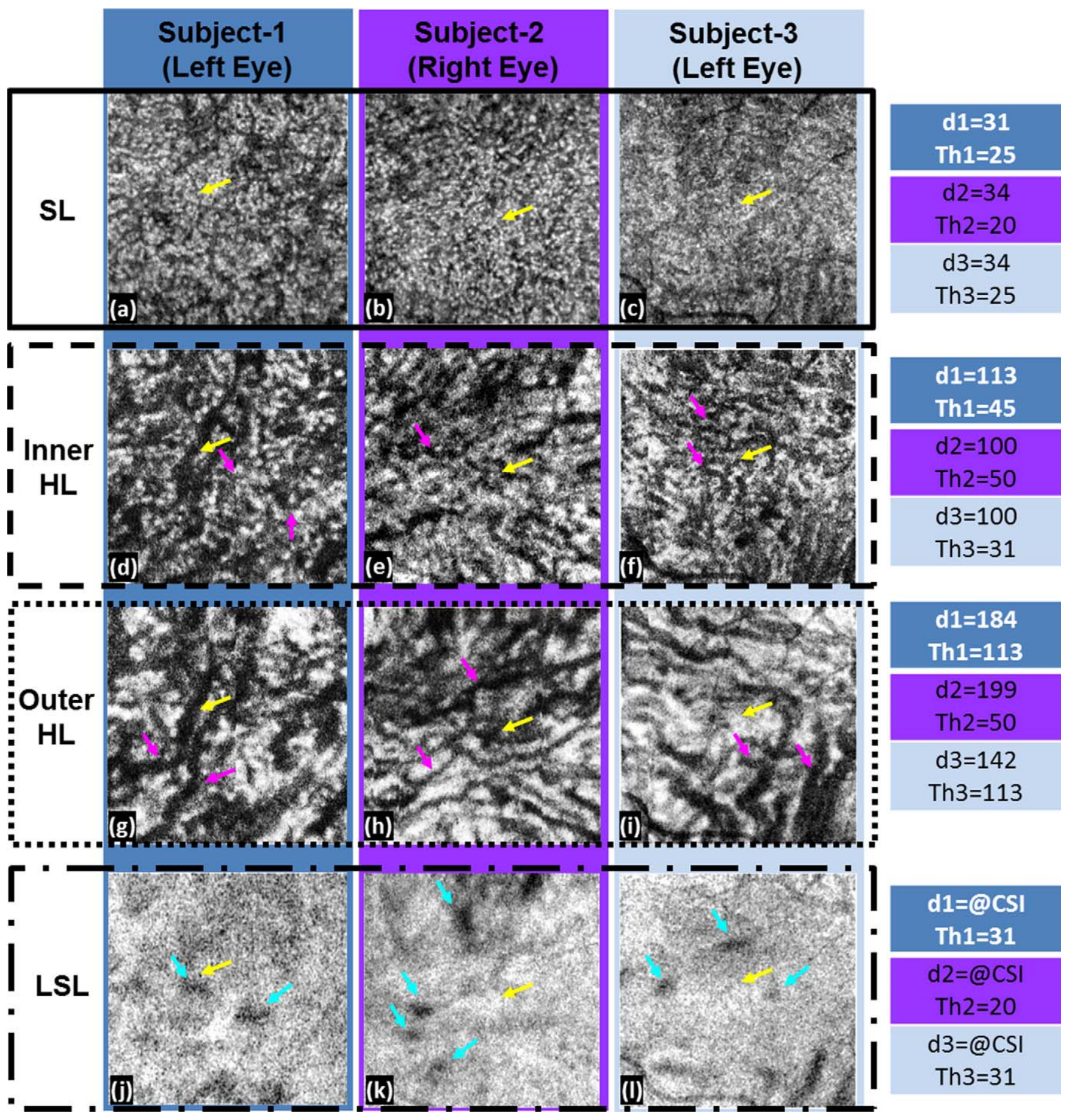

Figure 4. SS-OCT perifoveal en face views of the choroidal substructure and vasculature over $4 \times 4 \mathrm{~mm}$ in three healthy volunteers (a-1). (a-c) Dark and bright spotted appearances of the SL. (d-f) The inner HL with straight and tortuous vessels (magenta arrows). (g-i) The large lumen vessels (magenta arrows) coursing straightly in the outer HL. (j-1) The entrance sites of the temporal distal SPCAs (dark regions, cyan arrows) at the level of LSL. Yellow arrows indicate the fovea centralis projections at different layers. Subject 1: 28-year-old (left eye), subject 2: 37-year-old (right eye), and subject 3: 19-year-old (left eye). Thickness (Th) and depth (d) indicate the integration width and location of the en face view posterior to Bruch's membrane in micrometers for each subject. Indices determine the subject number. LSL was visualized as depth-integrated en face image, summing the reflectance signal over Th around the captured choroidal-scleral junction.

consisting of 907 depth scans over $5 \mathrm{~mm}$. This tomogram captures all major retina layers and the multiple layers of the choroidal region exhibiting between Bruch's membrane and lamina fusca sclerae (LFS). Different depth locations of the en face images are color-coded ${ }^{14}$ in Figure $2 b$ and shown en face in Figures 3-9. Sagittal (Figs. 2c-e) and transverse (Fig. 2f) tomograms are shown over $10 \mathrm{~mm}$ (907 depth scans) through the indicated different retinal slices. SS-OCT tomograms exhibit deep penetration at the $\mathrm{ONH}$, capturing the lamina cribrosa (LC) structure (white arrow, Fig. 2d). These tomograms depict retinal and choroidal thickness variations in different regions of the posterior segment as shown by Esmaeelpour et al. ${ }^{26}$ 

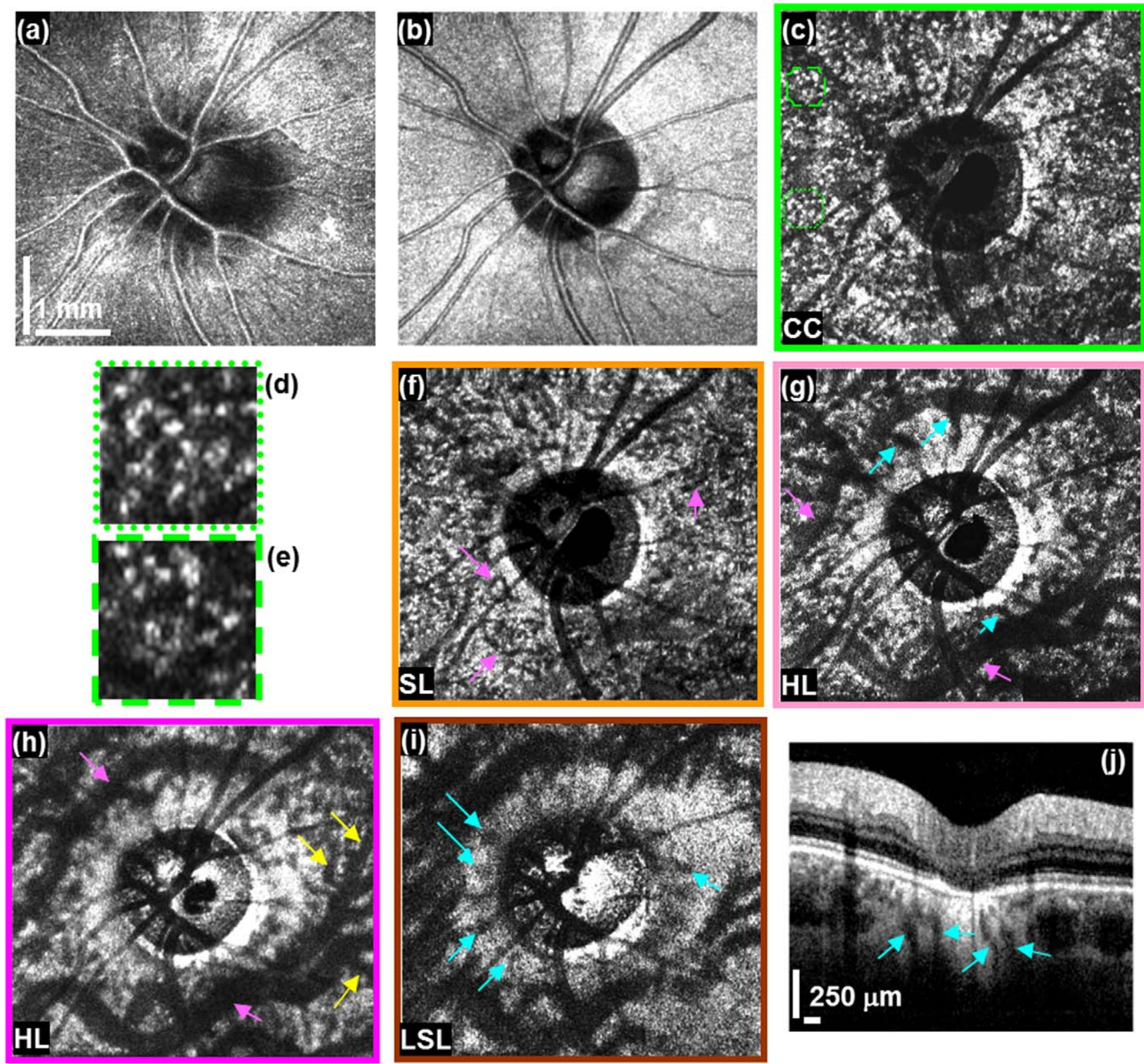

Figure 5. SS-OCT peripapillary en face views of the retina and choroid $(5 \times 5 \mathrm{~mm})(\mathbf{a}-\mathbf{i})$ and paraoptic SPCAs visualization in a SS-OCT 2D sagittal tomogram (j) in a 37-year-old normal subject's right eye. (a) Major arteries and veins in the en face view at the level of the RNFL. (b) The full-depthintegrated en face image capturing the shadow of the major vessels. (c) The choroidal en face view at the level of CC (thickness $=20 \mu \mathrm{m})$ around the papilla. (d-e) Two close-up views of the signified regions with dotted and dashed lines in (c). (f) The SL (thickness $=30 \mu \mathrm{m}$ ), unveiling small and tortuous vessels (magenta arrows). (g) The inner HL (thickness $=50 \mu \mathrm{m}$ ) and (h) the outer HL (thickness $=50 \mu \mathrm{m}$ ), capturing large choroidal vessels (magenta arrows) and their branches (yellow arrows) as well as paraoptic vessels extending radially toward the superior and inferior sectors (cyan arrows). (i) Twenty-micrometer-depth-integrated en face view at the level of the LSL showing the entrance sites of the paraoptic SPCAs (cyan arrows). (j) A sagittal tomogram depicting paraoptic SPCAs (cyan arrows) piercing the inner sclera and LSL.

\section{Retinal and Choroidal Vascular Pattern Visualization in the Macular Region}

Figures $3 \mathrm{a}-\mathrm{h}$ present en face views of the inner retina, the outer retina, and the choroid in the macular region. The superficial network of the blood vessels (Fig. 3a) supplying the inner retina layers is revealed within the ganglion cell layer (GCL) beneath the retina nerve fiber layer (RNFL), as is the capillary meshwork of the inner nuclear layer (INL). The inner/ outer photoreceptor segments (IS/OS) and the retina pigment epithelium (RPE) of the outer retina are captured in Figures 3b and $3 \mathrm{c}$. The fovea centralis (magenta arrow) and the shadow of the major retina vessels are depicted in the IS/OS en face image (Fig. 3b). The bright spotted region above the Bruch's membrane is the RPE cell layer (Fig. 3c). Multiple choroidal layers are evident deep to the RPE (Figs. 3d-h) between Bruch's membrane and LFS. These choroidal layers can be distinguished by their distinct melanocyte densities, vessel sizes, and shapes. ${ }^{14}$ It has been reported that the choroidal thickness not only differs between subjects but also varies for a given subject depending on the region of the fundus image. ${ }^{26}$ The total thickness of the choroidal layers was $\sim 300 \mu \mathrm{m}$ around the fovea centralis in this subject, in agreement with a previously reported thickness range. It has been reported that 

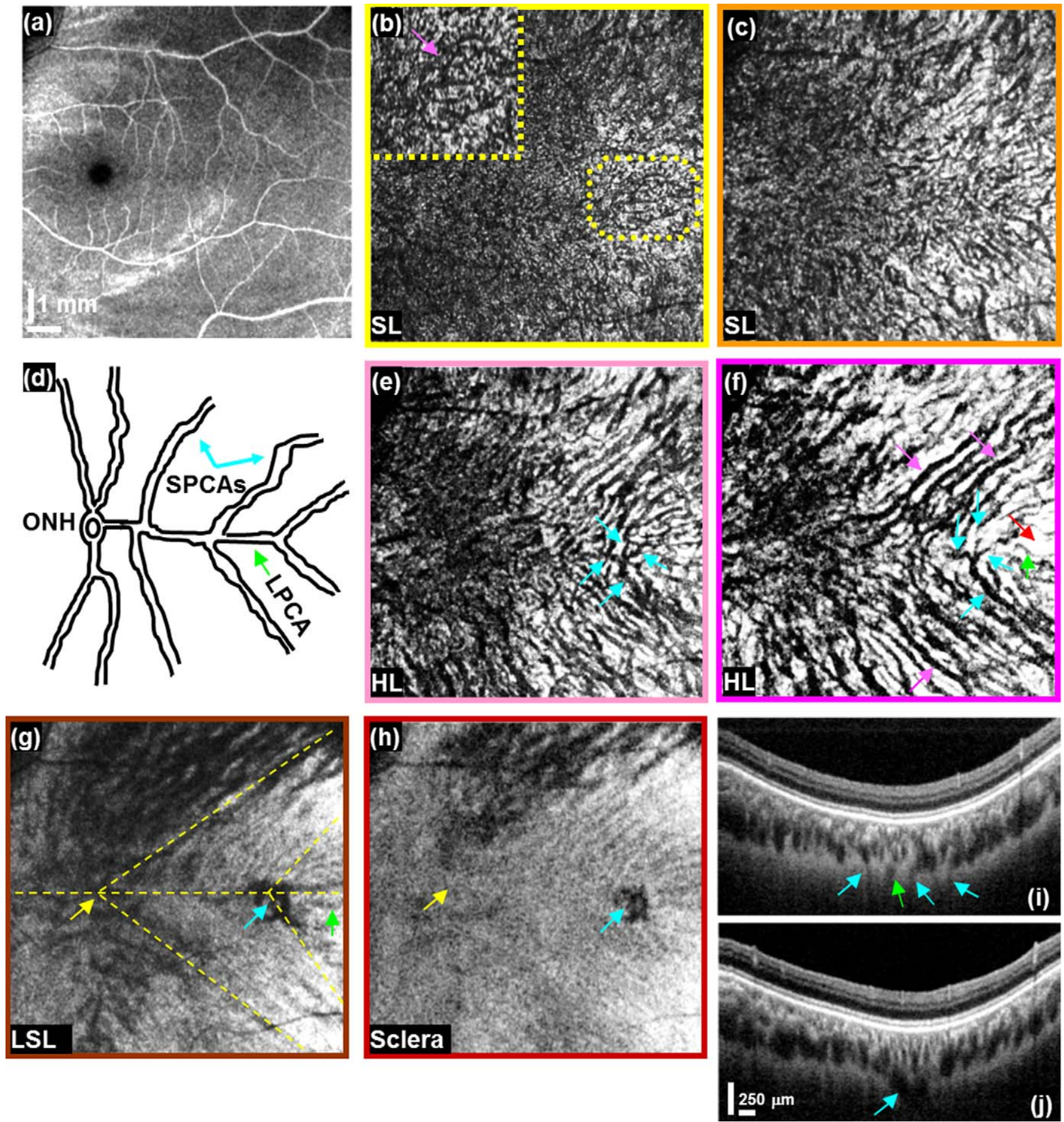

FiguRE 6. SS-OCT $10 \times 10 \mathrm{~mm}$ en face views of the retina, choroid, and sclera over a lateral sector of the ONH in a 37 -year-old normal subject's right eye. (a) The en face image depicts the major vessels (thickness $=100 \mu \mathrm{m}$ ). (b) The inner SL (thickness $=30 \mu \mathrm{m}$ ) image reveals the spindleshaped vessels (magenta arrow, the signified area with dotted line in b). (c) The en face image of the outer SL (thickness $=30 \mu \mathrm{m}$ ) captures its arteries and veins. (d) A schematic representation of the SPCAs and LPCA distribution in the HL as described by Hayreh. ${ }^{4}$ (e) The en face image of the inner HL (thickness $=50 \mu \mathrm{m}$ ) depicts the distal SPCAs originating from the temporal side (cyan arrows) of the fovea centralis. (f) The image of the outer HL (thickness $=40 \mu \mathrm{m}$ ) captures the superotemporal and inferotemporal vessels (magenta arrows), SPCA branches, and LPCA (cyan and green arrows). (g) Fifteen-micrometer-depth-integrated en face view at the level of LSL possibly reveals the lateral LPCA (green arrow). (h) The en face view of the inner sclera (thickness $=30 \mu \mathrm{m}$ ) showing a 1-mm diameter conduit (cyan arrow) comprised of an array of PCAs perforating the sclera. (i-j) Two sagittal tomograms depict the temporal SPCAs (cyan arrows) and lateral LPCA (green arrow) piercing the sclera and LSL. Yellow arrows $(\mathbf{g}, \mathbf{h})$ indicate the fovea centralis projections in the LSL and sclera en face views. Yellow dotted lines (g) present the PCAs and veins distribution in the HL as shown in (f). 4,30 

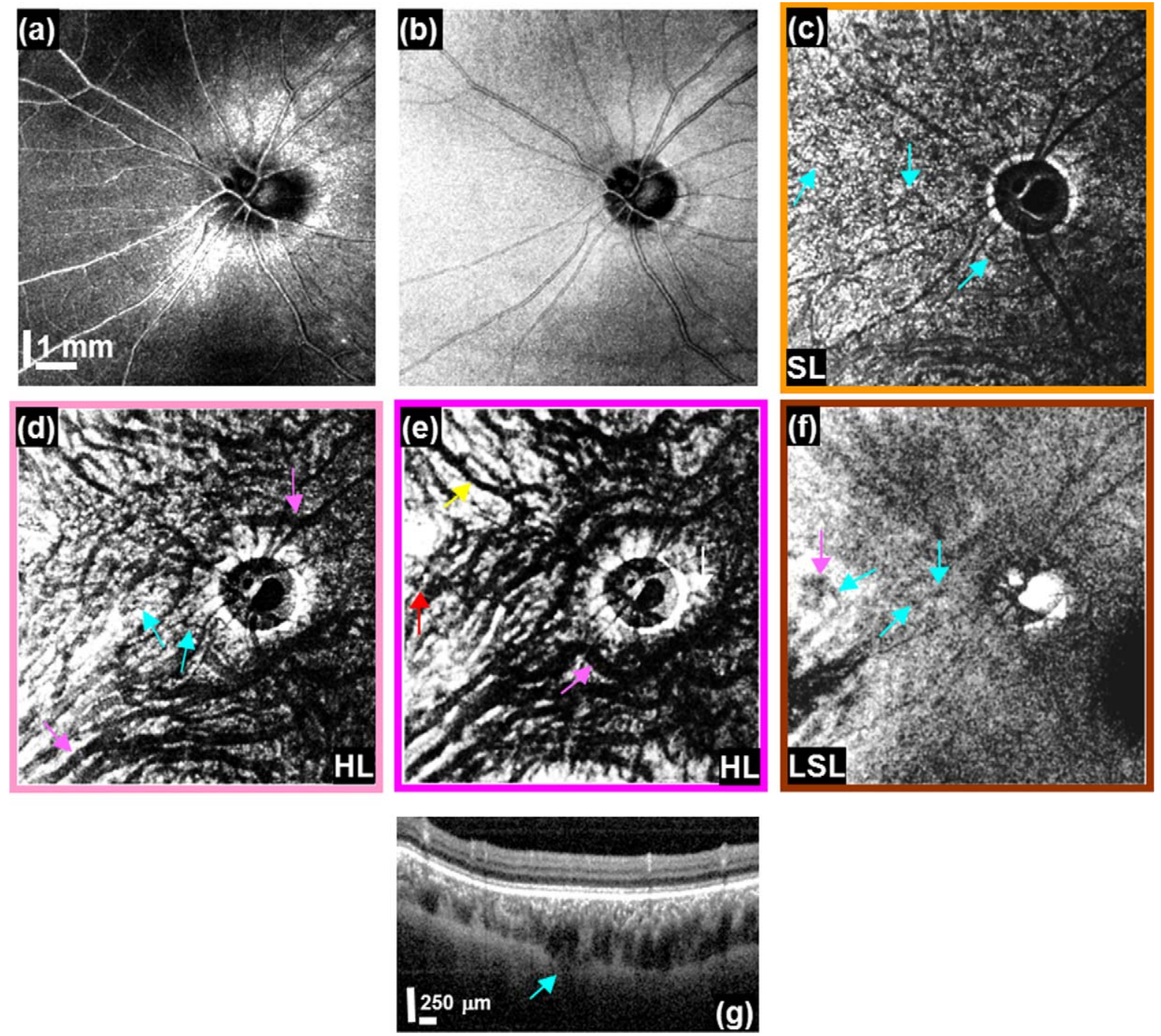

Figure 7. SS-OCT retinal and choroidal en face views over a $10 \times 10 \mathrm{~mm}$ medial sector of the fovea (a-f) and medial SPCA visualization in a SS-OCT 2D sagittal tomogram (g) in a 37-year-old normal subject's right eye. Major arteries and veins are depicted in (a) an en face view at the level of the RNFL (thickness $=50 \mu \mathrm{m}$ ) and (b) a full-depth-integrated en face image capturing the shadow of the major vessels. (c) The inner SL (thickness $=30$ $\mu \mathrm{m}$ ) contains small vessels (cyan arrows). (d) The inner HL (thickness $=50 \mu \mathrm{m}$ ) and (e) the outer HL (thickness $=40 \mu \mathrm{m}$ ) show large superonasal (yellow arrow), inferonasal vessels (magenta arrows), medium-sized vessels (cyan arrow), paraoptic PCA branch (white arrow), and LPCA (red arrow). (f) Fifteen-micrometer-depth-integrated en face view at the level of the LSL possibly reveals the entrance sites of the individual medial SPCAs (cyan arrows) and the medial PCA arrays (magenta arrow). (g) A sagittal tomogram that depicts the medial SPCA (cyan arrow).

the most superficial layer, choriocapillaris (CC), supplies blood to the outer retina layers and has a patch-like appearance with lobular structures. ${ }^{27}$ Each lobule consists of a central feeding arteriole and peripheral draining venuoles. ${ }^{27,28}$

Figure $3 \mathrm{~d}$ depicts dark and bright spots over a $1-\mathrm{mm}^{2}$ area beneath the RPE at the level of CC (signified in Fig. 3c). A similar spotted appearance of the CC structure has been observed using 1 micron SD-OCT. ${ }^{14}$ Our hypothesis is that the spots result from the size of the microcapillary arterioles and venuoles in the CC being smaller than our SS-OCT transverse resolution $(\sim 20 \mu \mathrm{m})$. Sattler's layer (SL) comprises melanocytes and medium-sized vessels (arterioles and venuoles). Arterioles originate from PCAs and extend vertically in the SL to supply the CC. Venuoles run obliquely to drain the CC and transport waste to the major veins. Arterioles and venuoles (dark spots) as well as melanocytes (bright spots) ${ }^{14}$ are depicted in the SL en face view (Fig. 3e). The Haller's layer (HL) contains large lumen vessels situated at the posterior part of the SL including PCA branches and major veins. These PCA branches supply the choroid up to the equator. ${ }^{29}$ Figure $3 f$ reveals straight and tortuous vessels (cyan arrows) in the inner HL. Several vessel branches (magenta arrows) coursing in the HL are shown in Figure $3 \mathrm{~g}$. Beneath the HL, the avascular lamina suprachoroid layer (LSL) contains entrance sites for the temporal distal short posterior ciliary arteries (SPCAs, cyan arrows; Fig. 3h). The temporal distal SPCA (cyan arrows) is seen perforating the sclera and choroid in the 2D tomogram presented in Figure $3 i$. Diameters of the temporal distal SPCA branches perforating the LSL range from 50 to $200 \mu \mathrm{m}$.

To evaluate the reproducibility of the choroidal structure and vasculature features captured in each layer, we acquired data over the macular regions of three healthy volunteers using SS-OCT with $15-\mu \mathrm{m}$ transverse resolution. Figures $4 \mathrm{a}-1$ depict the structure and vessels distribution captured in the SL, HL, and LSL of three healthy volunteers. Comparisons of en face images associated with each choroidal layer in Figures 4a-1 show that SS-OCT can capture the same features in each choroidal layer of different subjects. 


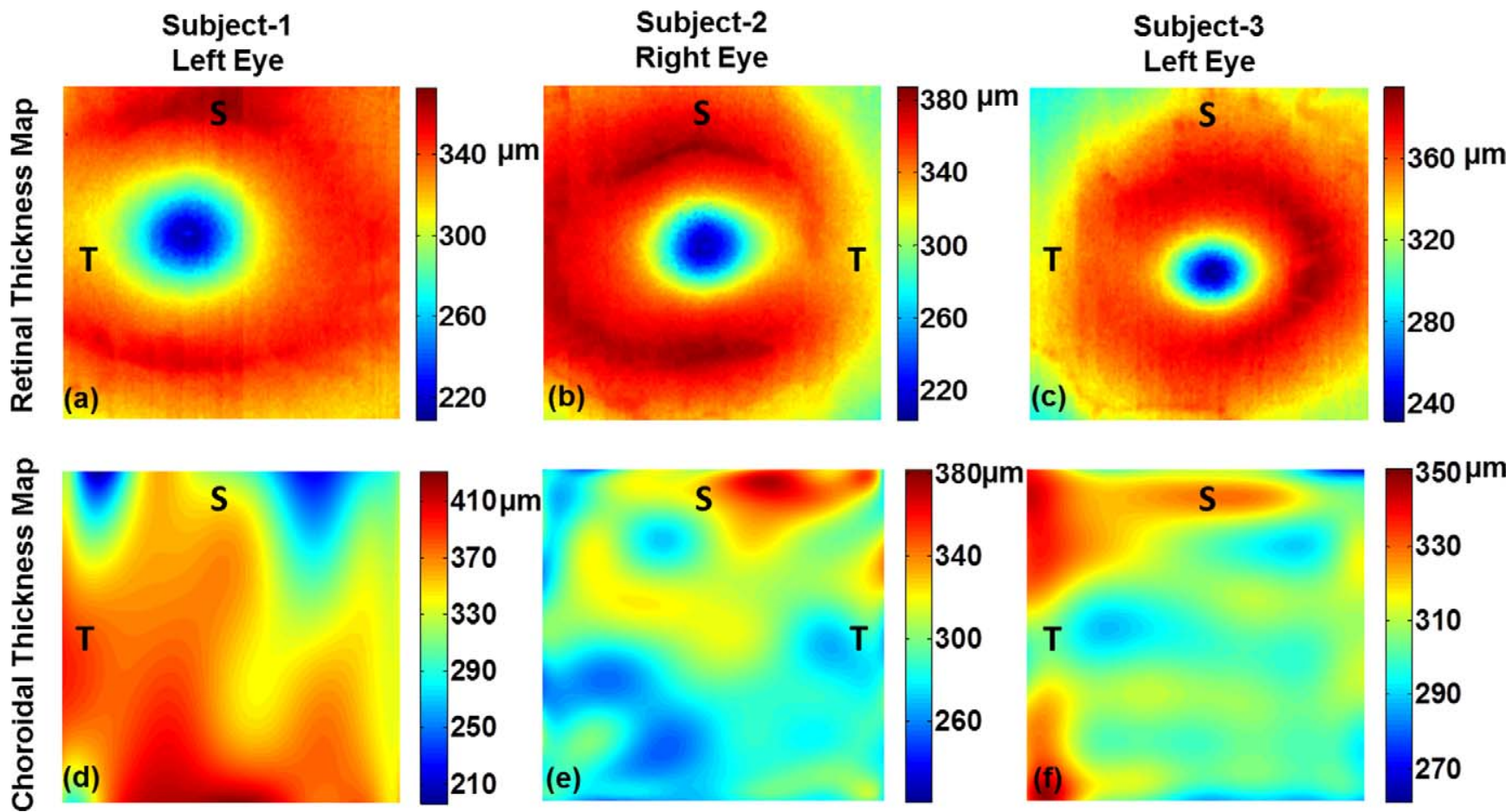

FigURE 8. SS-OCT retinal (a-c) and choroidal (d-f) thickness maps over $4 \times 4 \mathrm{~mm}$ of the macular region centered on the fovea in three healthy subjects. (a) Retinal and (d) choroidal thickness maps of a 28-year-old subject's left eye. (b) Retinal and (e) choroidal thickness maps of a 37-year-old subject's right eye. (c) Retinal and (f) choroidal thickness maps of a 19-year-old subject's left eye. S: Superior quadrant and T: temporal quadrant.

The same bright-dark spotted appearance is present in each SL en face view (Figs. $4 \mathrm{a}-\mathrm{c}$ ). Tortuous vessels (magenta arrows, Figs. 4d-f) are visualized in the inner HL. SS-OCT also captures large lumen vessels (magenta arrows, Figs. 4g-i) with different coursing directions in the outer HL of these volunteers, as previously visualized by ICGA. ${ }^{30}$ Entrance sites for the temporal distal SPCAs (dark regions, cyan arrows) piercing the LSL are depicted in Figures $4 \mathrm{j}-1$

\section{Peripapillary Vascular Pattern Imaging}

The branches of blood vessels supplying the inner retinal layers and running radially from $\mathrm{ONH}$ are presented in Figures $5 \mathrm{a}$ and 5 b. Figure $5 \mathrm{c}$ shows the choroidal en face view at the level of CC captured by SS-OCT. The spotted appearance in the signified areas of Figure 5c (zoom in Figs. 5d, 5e) is consistent with the insufficient SS-OCT transverse resolution $(\sim 20 \mu \mathrm{m})$ to capture the fine structures of $\mathrm{CC}$. The SL around the papilla (Fig. 5f) consists of small winding vessels (magenta arrows, Fig. 5f). The vessels coursing radially (cyan arrows) toward the superior and inferior sectors of the HL are depicted in Figure $5 \mathrm{~g}$. Large inferonasal vessels (magenta arrows, Figs. 5g, 5h) run around the $\mathrm{ONH}$ in the HL and start branching (yellow arrows, Fig. 5h). The entrance sites of the paraoptic SPCAs (cyan arrows) piercing the LSL around the papilla are shown in Figure 5i. Paraoptic SPCAs (cyan arrows) originate from the ophthalmic artery and pierce the choroid at the level of the LSL. Four of these paraoptic SPCAs are captured in a 2D tomogram through the $\mathrm{ONH}$ (Fig. $5 \mathrm{j}$ ).

\section{Wide-Angle Visualization of the Lateral Side of the ONH}

Wide-angle OCT imaging at $1 \mu \mathrm{m}$ provides a capability to capture and follow the retinal and choroidal structure and vasculature network over an extended area. Figures $6 a-h$ depict en face views of the retina and choroid over a $10 \times 10$ $\mathrm{mm}$ sector of the retina temporal to the ONH. The superficial network of the blood vessels supplying and draining capillaries in the inner retina layers is visualized in Figure 6a. The en face images of the choroid and sclera are shown in Figures $6 \mathrm{~b}-\mathrm{h}$. A fly-through of the wide-angle images (Figs. 6b-h) clearly depicts change in the vascular pattern and vessel density through the depth. Increase in the vessel size is observed through the choroidal depth (Figs. 6b-f). Spindle-shaped vessels (magenta arrow, the signified area of Fig. 6b) appear to perfuse and drain the CC. Figures $6 \mathrm{c}-\mathrm{f}$ visualize the density, direction, and thickness of the individual vessels in the SL and HL. Small vessels are seen in the inner SL (Fig. 6b) and larger straight vessels in the outer SL (Fig. 6c). Figure 6d depicts a schematic diagram representing the temporal SPCAs (cyan arrows) and lateral long PCA (LPCA, green arrow) distribution in the HL. ${ }^{4}$ Each of these PCAs extends straight from the posterior pole to the equator and supplies a sector of the choroid. ${ }^{29}$ The borders between these sectors demarcate the arterial watershed zones. ${ }^{4}$ Figures $6 \mathrm{e}$ and $6 \mathrm{f}$ reveal four temporal distal SPCA branches (cyan arrows) emerging from the temporal side of the fovea centralis and running straight in different directions. The superotemporal and inferotemporal major choroidal vessels (Fig. 6f, magenta arrows) are organized into a symmetric distribution of segmental arteries and veins in the lateral quadrants. 4,30 The maximum measured size of these vessels is $\sim 200 \mu \mathrm{m}$ in the HL. The thickness of these large HL veins and PCAs largely determines the choroidal thickness. The large entrance site (cyan arrow, Figs. $6 \mathrm{~g}, 6 \mathrm{~h}$ ) of the temporal distal SPCA arrays (cyan arrows, Fig. 6e) and LPCA (green arrow, Figs. 6f, 6g) perforating the sclera and LSL is captured for the first time using an OCT system. An array of PCAs enters the choroid in a 1-mm-diameter conduit (cyan arrow, Fig. 6h) and starts branching out $5 \mathrm{~mm}$ temporal and $780 \mu \mathrm{m}$ inferior of the fovea centralis (yellow arrow, Figs. $6 \mathrm{~g}, 6 \mathrm{~h}$ ). The lateral LPCA (green arrow, Figs. 6f, 6g) is seen coursing anteriorly 


\section{CC}
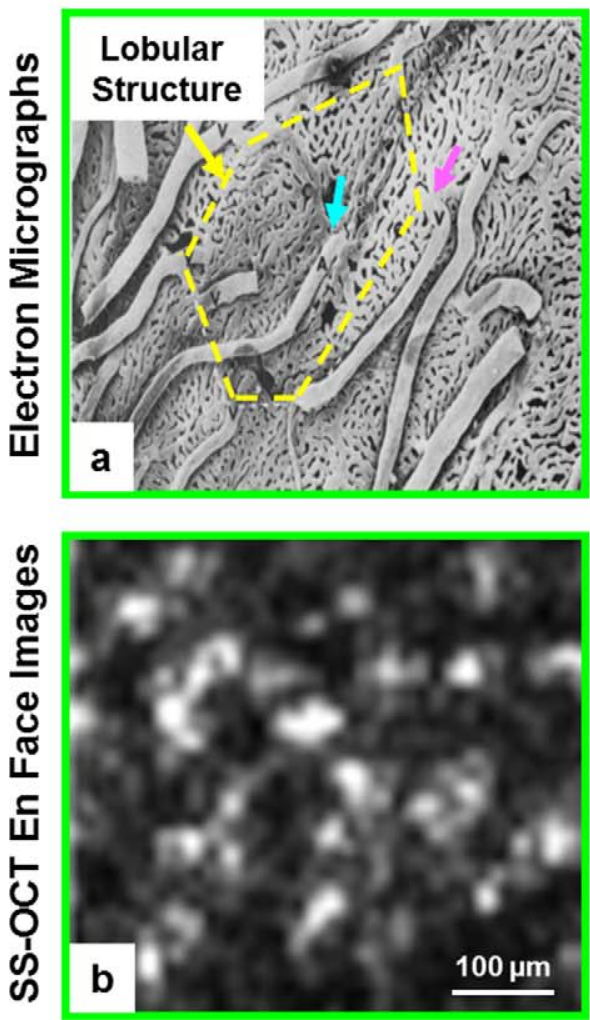

SL
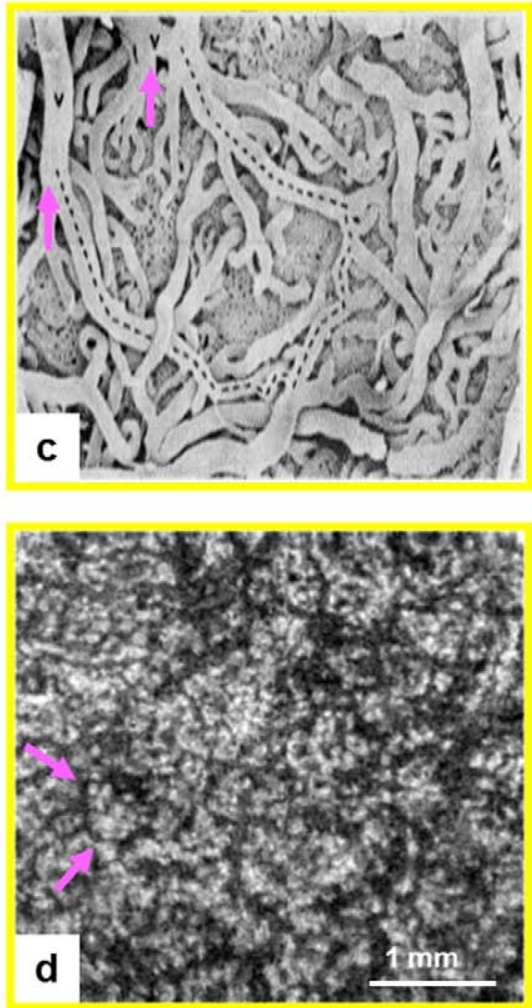

HL
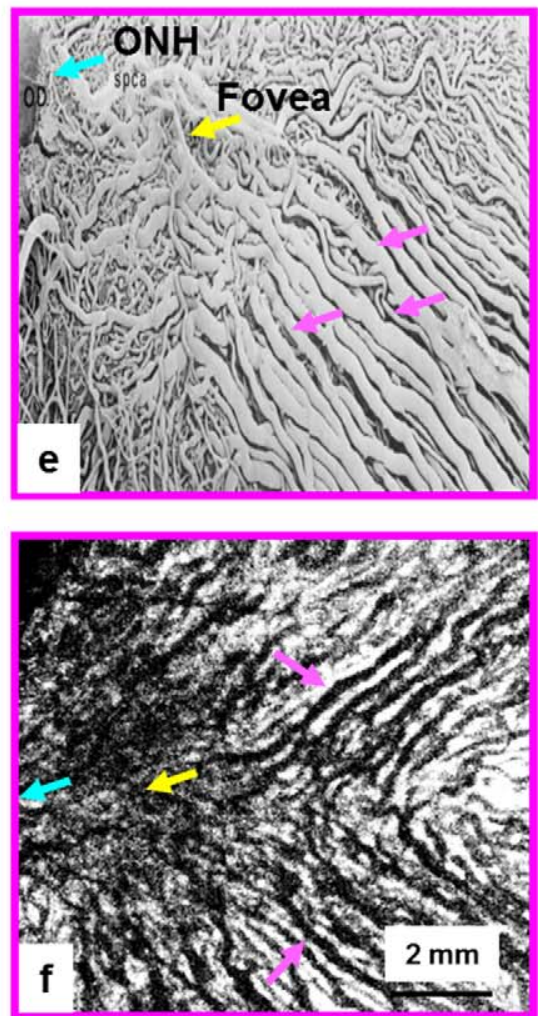

FIGURE 9. Scanning electron micrographs and SS-OCT reflectance en face images of human CC (a, b), SL (c, d), and HL (e, f). Scanning electron micrographs of (a) CC revealing lobular structure, arteriole (cyan arrow), and venuoles (magenta arrow), (c) SL unveiling medium-sized vessels (magenta arrows), and (e) HL visualizing large lumen veins and arteries (magenta arrows). Depth-integrated SS-OCT en face images at the level of (b) outer CC around papilla $(500 \times 500 \mu \mathrm{m})$, (d) SL capturing medium-sized vessels (magenta arrows) in the macular region $(4 \times 4 \mathrm{~mm})$, and $(\mathbf{f}) \mathrm{HL}$ visualizing large lumen vessels (magenta arrows) in the lateral side of ONH $(10 \times 10 \mathrm{~mm})$. Yellow and cyan arrows in $\mathbf{e}$ and $\mathbf{f}$ indicate foveal centralis projection and ONH border in the HL, respectively. Reprinted with permission from Yoneya S, Tso MO. Angioarchitecture of the human choroid. Arch Ophthalmol. 1987;105:681-687. Copyright (C) 1987 American Medical Association. All rights reserved.

along the horizontal meridian through the LSL, consistent with known anatomy. ${ }^{4,31,32}$ A decreased density of vessels and increased density of melanocytes (hyperreflective region) are observed on the temporal side of this conduit in the HL (red arrow, Fig. 6f). The artery and vein distributions (yellow dotted lines, Fig. 6g) are somewhat symmetrical to the lateral side of the fovea centralis. ${ }^{4,30}$ Several temporal SPCAs (cyan arrows) and LPCA (green arrow) perforating the LSL are visualized in $2 \mathrm{D}$ tomograms (Figs. 6i, 6j)

\section{Wide-Angle Visualization of the Medial Side of the Macula}

Retinal and choroidal en face views (Figs. 7a-f) depict the vascular pattern, vessel size, and density throughout depth over a $10 \times 10 \mathrm{~mm}$ region medial to the macula. The superficial network of the blood vessels is seen in the $100-\mu \mathrm{m}$ depth-integrated en face image as well as the full-depthintegrated en face images (Figs. $7 \mathrm{a}, 7 \mathrm{~b}$ ). Figure $7 \mathrm{c}$ shows the medium-sized arteries and veins (cyan arrows). The captured large HL vessels in the inferior side of the ONH (Fig. 7c) show that the thickness of the SL varies. Figure $7 d$ reveals vessels in the HL including: large lumen straight vessels (magenta arrows) turning around the $\mathrm{ONH}$ and medium-sized vessels (cyan arrows). Paraoptic ciliary artery branches (white arrow) extend radially in the HL for supplying arterioles (Fig. 7e). The superonasal (yellow arrow) and inferonasal (magenta arrow) major choroidal vessels running in the medial quadrants are shown in Figure $7 e . .^{30,4}$ An asymmetric vessels distribution is observed over the medial side of the ONH (Fig. 7e). ${ }^{30}$

Figure 7e appears to capture the medial LPCA (red arrow), running toward to the equator along the horizontal meridian. Figure $7 \mathrm{f}$ shows the entrance site of the medial SPCA and medial LPCA (magenta arrow) perforating the LSL, $5 \mathrm{~mm}$ medially of the papilla in an $800-\mu \mathrm{m}$ diameter conduit. Dark spots (cyan arrows, Fig. $7 \mathrm{f}$ ) depict piercing locations of several individual medial SPCAs in the LSL. ${ }^{4}$ The bright region around the medial LPCA entrance site (magenta arrow) may reveal an increased number or altered orientation of the melanocytes. The medial SPCA (cyan arrow) is visualized in a 2D sagittal tomogram (Fig. 7g).

\section{Retinal and Choroidal Thickness Map of the Macular Region}

Figures 8a-c show the retinal thickness maps in the macular regions of three healthy subjects. It is clear that retinal thicknesses range from 200 to $400 \mu \mathrm{m}$ in the macular regions. Figures $8 \mathrm{~b}$ and $8 \mathrm{c}$ show that the retinal thicknesses decrease in the temporal quadrant of the retina. Red dark regions around the fovea centralis (Figs. 8a-c) reveal an increase in the retinal thickness due to the accumulation of fiber bundles in the RNFL. Figures 8d-f depict choroidal thickness maps over the same region. Maximum choroidal thickness ranges from 350 to 430 $\mu \mathrm{m}$ in these subjects. While the retinal thickness maps follow a cohesive pattern in these subjects, the choroidal thickness map 
varies substantially. The major HL vein and artery thicknesses are dominant in determining the choroidal thickness.

To study the repeatability of retinal and choroidal thickness measurements in a normal subject, OCT data sets were acquired over the macular region of the second subject's right eye $(4 \times 4 \mathrm{~mm})$ at four different time points in 1 hour. Standard deviations of retinal and choroidal thicknesses were less than 6 and $15 \mu \mathrm{m}(1.5 \%$ and $4 \%)$ for all obtained depth scans, respectively. Higher choroidal thickness measurement error is probably due to the lower signal-to-noise ratio in the outer choroid, which limits the segmentation algorithm for detecting the choroidal-scleral junction.

\section{Comparisons of Choroidal SS-OCT Images and Scanning Electron Micrographs}

In order to correlate captured SS-OCT en face images with known anatomy in the CC, SL, and HL, several scanning electron micrographs (Figs. 9a, 9c, 9e) are compared with SsOCT images (Figs. 9b, 9d, 9f). Figure 9a shows the electron micrograph of the posterior section of the CC. The yellow dashed line depicts a lobular structure (Fig. 9a). The feeding arteriole (cyan arrow) and draining venuole (magenta arrow) are easily distinguished in this image. Figure $9 \mathrm{~b}$ depicts an SSOCT en face image at the level of the outer CC. Comparisons of Figures $9 \mathrm{a}$ and $9 \mathrm{~b}$ show that the size of features such as microcapillaries as well as arteriole and venuoles in the $\mathrm{CC}$ are smaller than our SS-OCT system transverse resolution $(\sim 15$ $\mu \mathrm{m}$ ) to be captured. The SL scanning electron micrograph (Fig. 9c) depicts small vessels, arterioles, and venuoles (magenta arrows) running in the SL and extending to the $\mathrm{CC}$ after dissecting large vessels in the HL. SS-OCT en face image at the level of SL captures dark and bright regions (Fig. 9d). Comparisons of Figures 9c and 9d might associate dark regions and spots with medium-sized vessels (small vessels, arteriole, and venuoles, magenta arrows) coursing in the SL and approaching vertically and obliquely to the CC. Bright spots can be interpreted as SL melanocytes in the SS-OCT en face image due to their higher reflection. A scanning electron micrograph of the HL (Fig. 9e) shows that the large lumen vessels (magenta arrows) are arranged in nearly straight paths in this layer. Cyan and yellow arrows indicate $\mathrm{ONH}$ border and fovea centralis projection in the HL, respectively. HL vessels (magenta arrows) can simply be correlated by comparing HL electron micrograph (Fig. 9e) and captured SS-OCT images at the level of the HL (Fig. 9f).

\section{Discussion}

Although ICGA angiography has emerged as an important tool for inner and outer choroidal vascular visualization, it has two limitations: it requires dye injection, which makes it an invasive technology, and it lacks depth information. While the higherspeed line-scan CCD cameras that are needed for SD-OCT at 1 micron are becoming available, the superior detection efficiency and sensitivity roll-off performance of SS-OCT make it more attractive than SD-OCT for choroidal visualization at very high speeds. Retina and $\mathrm{ONH}$ en face imaging ${ }^{19}$ as well as choroidal thickness measurement were demonstrated in normal subjects using SS-OCT ${ }^{33}$ and SD-OCT. ${ }^{34}$ In our study, the ability of wide-angle SS-OCT at $1060 \mathrm{~nm}$ for individual retinal and choroidal layer visualization was demonstrated at high resolution. Our results show that SS-OCT centered on the 1060-nm water transmission window enables visualization of the vessel distribution, size, and density through the different choroidal layers. The fly-through of the images depicts increases in the choroidal vessel size through the depth. This noninvasive technology is also able to assess major draining veins and PCA branches in en face images throughout the depth and capture their course toward the equator. Wide-angle SS-OCT with enhanced sensitivity and penetration depth enables us to visualize PCA entrance sites in the temporal and medial sides of $\mathrm{ONH}$ at the inner sclera level. SS-OCT shows its ability to capture symmetric and asymmetric artery and vein distributions in the lateral and medial quadrants as previously visualized by ICGA. ${ }^{30}$ Retinal and choroidal thicknesses can be measured by using SS-OCT at 1 micron. Dense sampling approaches and averaging methods over a small transverse region might capture variations in the choroidal thickness maps (Figs. 8d-f) caused by the choroidal vessels at the level of the choroidal-scleral junction, which were indiscernible in the previous studies. ${ }^{33,34}$ The same captured features in each choroidal layer of three different healthy volunteers might demonstrate the reproducibility of SSOCT measurements. One micron SS-OCT choroidal imaging of additional normal individuals can provide a basis for comparison to choroidal alterations that may occur in common diseases such as AMD, degenerative myopia, and even, potentially, some forms of glaucoma. These alterations can be detected by monitoring morphological metrics and maps such as choroidal layer thickness, vessel distribution, density, and size, as well as PCA entrances. Combination of morphological structure and phase information ${ }^{35}$ might also enhance retinal and choroidal microvasculature visualization. While we demonstrated SS-OCT at 1 micron as a powerful tool for choroidal vascular imaging, there is still a need for robust methods to noninvasively differentiate choroidal arteries from veins.

\section{Acknowledgments}

The authors thank Carl Zeiss Meditec for providing the patient interface part of their Stratus OCT system.

\section{References}

1. Friedman E. A hemodynamic model of the pathogenesis of agerelated macular degeneration. Am J Ophthalmol. 1997;124: 677-682.

2. Flammer J, Orgul S, Costa VP, et al. The impact of ocular blood flow in glaucoma. Prog Retinal Eye Res. 2002;21:359-393.

3. Hayreh SS. Anterior Ischemic Optic Neuropathy. Heidelberg, Germany: Springer-Verlag; 1975.

4. Hayreh SS. Posterior ciliary artery circulation in health and disease: the Weisenfeld lecture. Invest Ophthalmol Vis Sci. 2004;45:749-757.

5. Gass JDM. Stereoscopic Atlas of Macular Diseases. 4th ed. St. Louis, MO: Mosby; 1997.

6. Yannuzzi LA, Rohrer KT, Tindel LJ, et al. Fluorescein angiography complication survey. Ophthalmology. 1986;93: 611-617.

7. Hope-Ross M, Yannuzzi LA, Gragoudas ES, et al. Adverse reactions due to indocyanine green. Ophthalmology. 1994; 101:529-533.

8. Wojtkowski M, Bajraszewski T, Targowski P, Kowalczyk A. Realtime in vivo imaging by high-speed spectral optical coherence tomography. Opt Lett. 2003;28:1745-1747.

9. Nassif NA, Cense B, Park BH, et al. In vivo high-resolution video-rate spectral-domain optical coherence tomography of the human retina and optic nerve. Opt Express. 2004;12:367376.

10. Anger EM, Unterhuber A, Hermann B, et al. Ultrahigh resolution optical coherence tomography of the monkey fovea: identification of retinal sublayers by correlation with semithin histology sections. Exp Eye Res. 2004;78:1117-1125. 
11. Gloesmann M, Hermann B, Schubert C, Sattmann H, Ahnelt PK, Drexler W. Histologic correlation of pig retina radial stratification with ultrahigh-resolution optical coherence tomography. Invest Ophthalmol Vis Sci. 2003;44:1696-1703.

12. Unterhuber A, Považay B, Hermann B, Sattmann H, ChavezPirson A, Drexler W. In vivo retinal optical coherence tomography at $1040 \mathrm{~nm}$-enhanced penetration into the choroid. Opt Express. 2005;13:3252-3258.

13. Považay B, Bizheva K, Hermann B, et al. Enhanced visualization of choroidal vessels using ultrahigh resolution ophthalmic OCT at $1050 \mathrm{~nm}$. Opt Express. 2003;11:1980-1986.

14. Považay B, Hermann B, Hofer B, et al. Wide-field optical coherence tomography of the choroid in vivo. Invest Ophthalmol Vis Sci. 2009;50:1856-1863.

15. Chinn SR, Swanson EA, Fujimoto JG. Optical coherence tomography using a frequency-tunable optical source. Opt Lett. 1997;22:340-342.

16. Potsaid B, Baumann B, Huang D, et al. Ultrahigh speed $1050 \mathrm{~nm}$ swept source/Fourier domain OCT retinal and anterior segment imaging at 100,000 to 400,000 axial scans per second. Opt Express. 2010;18:20029-20048.

17. Lee ECW, de Boer JF, Mujat M, Lim H, Yun SH. In vivo optical frequency domain imaging of human retina and choroid. Opt Express. 2006;14:4403-4411.

18. Yasuno Y, Hong YJ, Makita S, et al. In vivo high-contrast imaging of deep posterior eye by $1-\mu \mathrm{m}$ swept source optical coherence tomography and scattering optical coherence angiography. Opt Express. 2007;15:6121-6139.

19. Srinivasan VJ, Adler DC, Chen Y, et al. Ultrahigh-speed optical coherence tomography for three-dimensional and en face imaging of the retina and optic nerve head. Invest Ophthalmol Vis Sci. 2008;49:5103-5110.

20. Yun SH, Boudoux C, Tearney GJ, Bouma BE. High-speed wavelength-swept semiconductor laser with a polygon-scanner-based wavelength filter. Opt Lett. 2003;28:1981-1983.

21. ANSI. Safe Use of Lasers and Safe Use of Optical Fiber Communications. Orlando, FL: American National Standard Institute Z136 Committee; 2000:168

22. International Commission on Non-Ionizing Radiation Protection (ICNIRP). Revision of guidelines on limits of exposure to laser radiation of wavelengths between $400 \mathrm{~nm}$ and $1.4 \mu \mathrm{m}$. Health Phys. 2000;79:431-440.
23. Yasuno Y, Madjarova VD, Makita S, et al. Three-dimensional and high-speed swept-source optical coherence tomography for in vivo investigation of human anterior eye segments. $O p t$ Express. 2005;13:10652-10664.

24. Cense B, Nassif NA, Chen TC, et al. Ultra-high resolution high speed retinal imaging using spectral-domain optical coherence tomography. Opt Express. 2004;12:2435-2447.

25. Makita S, Hong Y, Yamanari M, Yatagai T, Yasuno Y. Optical coherence angiography. Opt Express. 2006;14:7821-7840.

26. Esmaeelpour M, Povazay B, Hermann B, et al. Threedimensional 1060-nm OCT: choroidal thickness maps in normal subjects and improved posterior segment visualization in cataract patients. Invest Ophthalmol Vis Sci. 2010;51:52605266.

27. Hayreh Ss. The choriocapillaris. Graefes Arch Clin Exp Ophthalmol. 1974;192:165-179.

28. Zhang HR. Scanning electron-microscopic study of corrosion casts on retinal and choroidal angioarchitecture in man and animals. Prog Retinal Eye Res. 1994;13:243-270.

29. Hayreh SS. Submacular choroidal vascular pattern: experimental fluorescein fundus angiographic studies. Graefes Arch Clin Exp Ophthalmol. 1974;192:181-196.

30. Mori K, Gehlbach PL, Yoneya S, Shimizu K. Asymmetry of choroidal venous vascular patterns in the human eye. Ophthalmology. 2004;111:507-512.

31. Risco JM, Grimson BS, Johnson PT. Angioarchitecture of the ciliary artery circulation of the posterior pole. Arch Ophthalmol. 1981;99:864-868.

32. Hayreh SS. The long posterior ciliary arteries: an experimental study. Graefes Arch Clin Exp Ophthalmol. 1974;192:197-213.

33. Hirata M, Tsujikawa A, Matsumoto A, et al. Macular choroidal thickness and volume in normal subjects measured by sweptsource optical coherence tomography. Invest Ophthalmol Vis Sci. 2011;52:4971-4978.

34. Ouyang Y, Heussen FM, Mokwa N, et al. Spatial distribution of posterior pole choroidal thickness by spectral domain optical coherence tomography. Invest Ophthalmol Vis Sci. 2011;52: 7019-7026.

35. Fingler J, Zawadzki RJ, Werner JS, Schwartz D, Fraser SE. Volumetric microvascular imaging of human retina using optical coherence tomography with a novel motion contrast technique. Opt Express. 2009;17:22190-22200. 